\title{
Compound screening platform using human induced pluripotent stem cells to identify small molecules that promote chondrogenesis
}

\author{
Sheng-Lian Yang ${ }^{1}$, Erica Harnish ${ }^{2}$, Thomas Leeuw ${ }^{3}$, Uwe Dietz ${ }^{3}$, Erika Batchelder ${ }^{1}$, Paul S. Wright ${ }^{2}$, \\ Jane Peppard ${ }^{2}$, Paul August ${ }^{2}$, Cecile Volle-Challier ${ }^{4}$, Francoise Bono ${ }^{4}$, Jean-Marc Herbert ${ }^{4}$, \\ Juan Carlos Izpisua Belmonte ${ }^{1,5 \bowtie}$ \\ ${ }^{1}$ Gene Expression Laboratories, The Salk Institute for Biological Studies, 10010 N. Torrey Pines Rd. La Jolla, CA 92037, USA \\ 2 Sanofi US, R\&D, Early to Candidate Unit, Tucson Research Center, 2090 E. Innovation Park Drive, Tucson, AZ 85755 , USA \\ ${ }^{3}$ Sanofi Deutschland GmbH, R\&D, TSU Aging Quality of Life, Industriepark Hoechst, Frankfurt am Main 65926, Germany \\ ${ }^{4}$ Sanofi R\&D, Early to Candidate Unit, 195, Route d'Espagne-BP 13669, Toulouse, France \\ ${ }^{5}$ Center of Regeneration Medicine, Dr. Aiguader 88, Barcelona 08003, Spain \\ $\triangle$ Correspondence: belmonte@salk.edu \\ Received October 24, 2010 Accepted October 28, 2010
}

\section{ABSTRACT}

Articular cartilage, which is mainly composed of collagen II, enables smooth skeletal movement. Degeneration of collagen II can be caused by various events, such as injury, but degeneration especially increases over the course of normal aging. Unfortunately, the body does not fully repair itself from this type of degeneration, resulting in impaired movement. Microfracture, an articular cartilage repair surgical technique, has been commonly used in the clinic to induce the repair of tissue at damage sites. Mesenchymal stem cells (MSC) have also been used as cell therapy to repair degenerated cartilage. However, the therapeutic outcomes of all these techniques vary in different patients depending on their age, health, lesion size and the extent of damage to the cartilage. The repairing tissues either form fibrocartilage or go into a hypertrophic stage, both of which do not reproduce the equivalent functionality of endogenous hyaline cartilage. One of the reasons for this is inefficient chondrogenesis by endogenous and exogenous MSC. Drugs that promote chondrogenesis could be used to induce self-repair of damaged cartilage as a non-invasive approach alone, or combined with other techniques to greatly assist the therapeutic outcomes. The recent development of human induced pluripotent stem cell (iPSCs), which are able to self-renew and differentiate into multiple cell types, provides a potentially valuable cell resource for drug screening in a "more relevant" cell type. Here we report a screening platform using human iPSCs in a multi-well plate format to identify compounds that could promote chondrogenesis.

KEYWORDS hESC, hiPSC, chondrogenesis, compound screening platform

\section{INTRODUCTION}

Cartilage is a stiff but flexible connective tissue that is mainly composed of chondrocytes in a matrix of type II collagen, proteoglycans and elastin fibers (Kuettner, 1992). Osteoarthritis (OA), a form of degenerative joint disease, is a painful deterioration of articular cartilage (Goldring and Goldring, 2007). The causes of OA include aging, obesity, acute injury and chronic overuse. Although OA is not life threatening, it does severely affect the quality of life of patients. OA is projected to become the fourth leading cause of disablement in the world by 2020 (Woolf and Pfleger, 2003). Due to the intricate and complex structure of articular cartilage, it is not able to fully self-repair after damage. Current drugs for OA just relieve the symptoms rather than cure the 
disease, and eventually almost all patients need joint replacement (Lohmander and Roos, 2007). Some approaches to repair the damaged cartilage are starting to become available, for example, microfracture, a modified technique of Pridie drilling, has been used in the clinic to induce the formation of fibrocartilaginous repair tissue by bone marrow cells infiltrating underneath the site of damage (Pridie, 1955; Smith et al., 2005). There are various types of MSCs such as bone marrow MSCs and adipose-derived MSCs that can differentiate into cartilage-like tissue in vitro and in vivo (Oreffo et al., 2005; Chanda et al., 2010), and these cell types have been recently suggested to be used as cellular therapy to repair degenerated cartilage (Falanga et al., 2007; Bulman et al., 2012). However, similar to joint replacement, both microfracture and MSC transplantation require expensive and somewhat invasive surgeries, and weeks to months for recovery. The clinical outcomes are affected by the age and health situation of the patients and size and stage of the cartilage lesions (William and Harnly, 2007). In addition, the repaired tissues formed by endogenous and exogenous MSCs are different from hyaline cartilage.

It has been shown that there are postnatal stem cells in healthy cartilage (Dowthwaite et al., 2004), and migratory chondrogenic progenitor cells in the repair tissue in human OA (Koelling et al., 2009). Drugs that induce self-repair of damaged cartilage with a non-intrusive approach alone or combined with microfracture and MSCs transplantation would have the potential to greatly facilitate cartilage repair (Yang et al., 2011). Our purpose, therefore, was to set up a screening platform to identify compounds that promote or induce chondrogenesis.

Reprogramming of human somatic cells back into a pluripotent state was first achieved through the expression of only four factors (Takahashi et al., 2007; Yu et al., 2007). Human iPSCs are similar to human embryonic stem cells in their ability to self-renew and differentiate into multiple cell types. Human iPSCs not only circumvent the ethical issues surrounding the use of embryos, but they can also be used to examine a variety of disease phenotypes. Because of these advantages, human iPSCs have been broadly used in basic research, disease modeling and have also been suggested to be used in cell therapy and drug screening (Ellis and Bhatia, 2011; Inoue and Yamanaka, 2011). Although differentiation of human iPSCs/hESCs into chondrocytes has been reported, those protocols are time-consuming and labor intensive, requiring over 4 weeks differentiation involving embryoid body (EB) formation and micro-mass cell pellet/high density cell mass (HDCM) culture (Koay et al., 2007; Toh et al., 2007). These limitations impede the development of a reproducible and small scale assay using iPSCs with which to screen compounds. Here we report a platform to identify compounds that promote chondrogenesis using human iPSCs as a source of cells. In this system, we have generated luciferase reporter lines from iPSCs and directly differentiated these reporter lines into chondrocytes without requiring 3D culture. We have further adapted this system into a 96-well plate format and validated our screening system using two novel synthesized chimeric peptides. Altogether, we have successfully established a 96-well screening platform for chondrogenesis using human IPSC that may be adapted to identify novel compounds that facilitate chondrocyte differentiation.

\section{RESULTS}

\section{Establishing and validating chondrogenic reporter lines}

iPSCs generated from human keratinocytes (KiPSCs) by retroviral transduction of the factors OCT4, SOX2, KLF4 and MYC were used in our studies (Aasen et al., 2008). Since collagen II is the most important and specific component in the extracellular matrix of cartilage (Kuettner, 1992), we chose collagen II as our chondrocyte marker, and established reporter lines that could represent endogenous collagen II expression levels. SOX9 is a SRY-related high mobility group (HMG) domain transcription factor and a master gene in chondrogenesis during development. There is a 48-bp enhancer sequence located in the col2a1 intron1 that is responsible for SOX9 binding and regulation of collagen II expression in vivo (Zhou et al., 1998). We cloned eight copies of this 48-bp SOX9 enhancer sequence upstream of a SV40 promoter driving luciferase as part of our expression cassette. The cassette also incorporated puromycin resistance as a positive selection strategy. It has been reported that hESCs can readily silence exogenous gene expression (Eiges et al., 2001; Liew et al., 2007). Chicken $\beta$-globin insulators have been shown to prevent this kind of silencing and have been suggested for use in gene therapy (Steinwaerder and Lieber, 2000; MacArthur et al., 2012). Since human iPSCs share similarities with hESCs, to ensure our expression cassette would not be silenced during passage and differentiation, we flanked our expression cassette with two copies of the core region of chicken $\beta$-globin insulators, on both sides (Fig. 1A). We then cloned the whole fragment into a lentiviral vector.

KiPSCs were infected with lentivirus and positive clones selected with puromycin. We then picked 12 single colonies to perform genomic PCR to confirm the integration of our expression cassette. As shown in Fig. 1B, all 12 colonies and the pools of the colonies demonstrate genomic insertions of the expression cassette, with varying numbers of integrations. We picked the clone that had the highest number of integrations (KiPSCs-SL) for the remaining assays. Examining the expression levels of the pluripotent markers TRA-1-81 and TRA-1-60 in KiPSCs-SL demonstrated that they retained levels comparable to those found in KiPSCs (Fig. 1C). To validate the chondrogenic differentiation ability of KiPSCs-SL, we performed differentiation experiments under TGF $\beta$ 
A

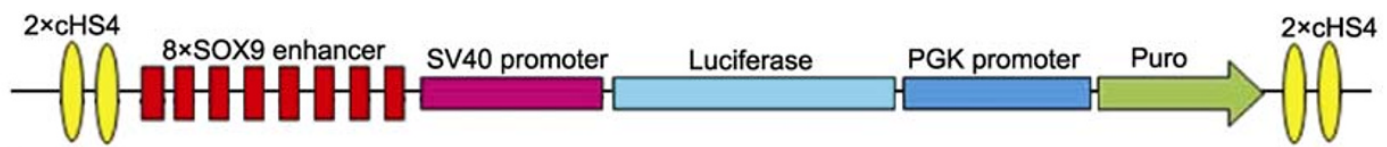

B
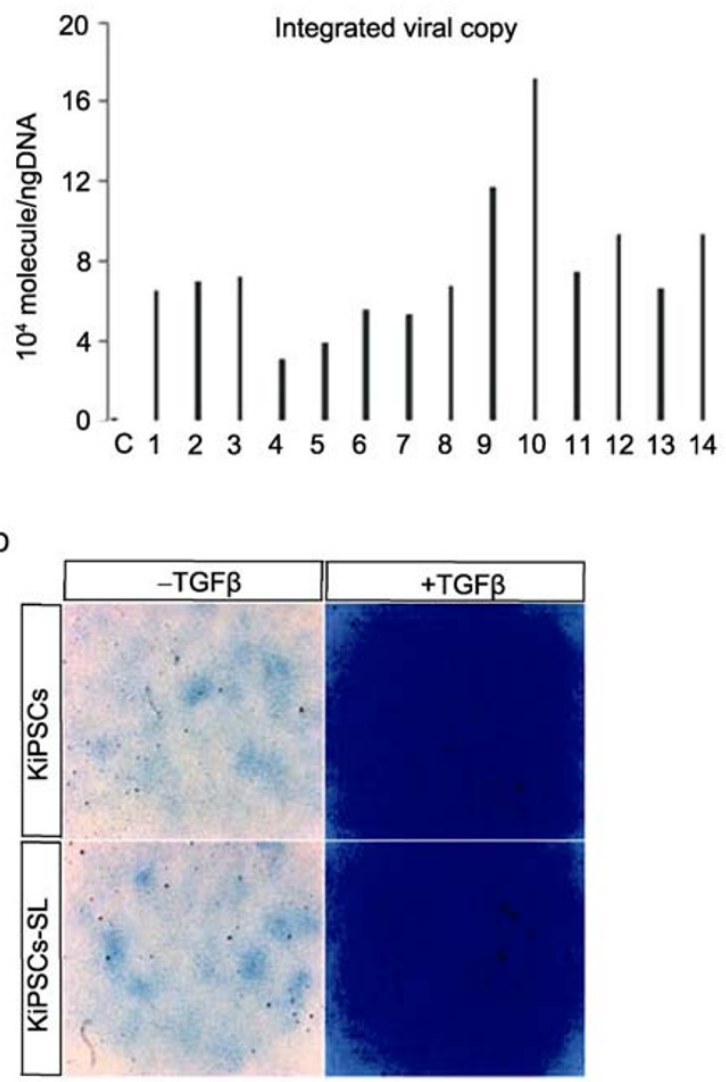

E

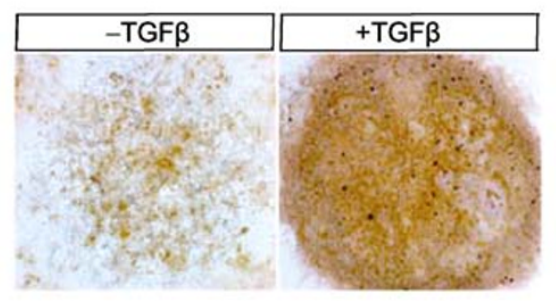

C

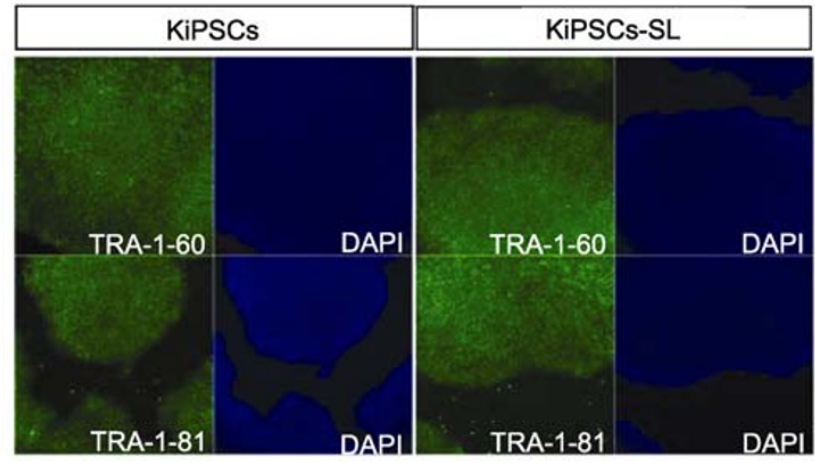

$\mathrm{F}$
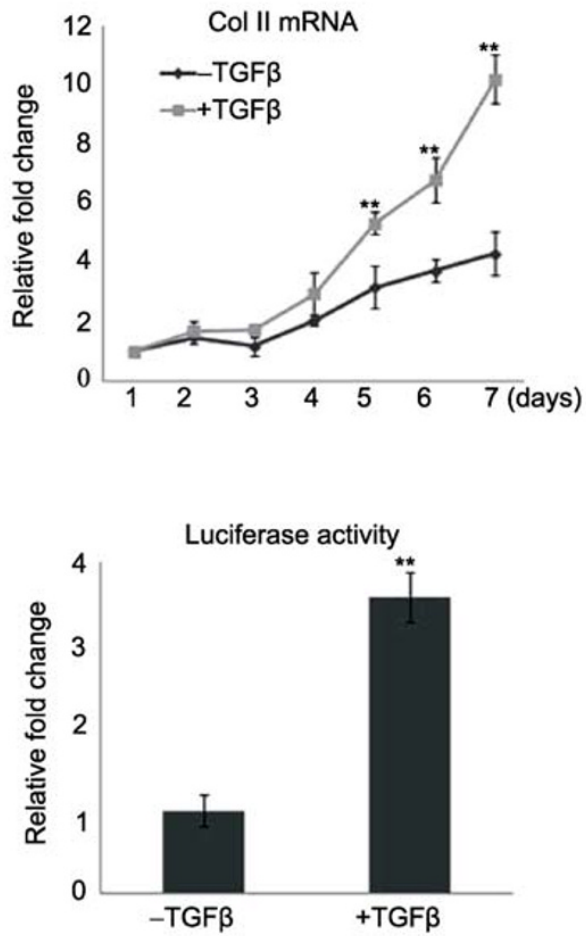

Figure 1. Establishing and validating chondrogenic reporter lines. (A) Diagram of expression cassette. cHS4: core region of chicken $\beta$-globin insulator; Puro: puromycin resistant gene. (B) Copy numbers of integrated viral vectors in the chromosomes of reporter lines as determined by genomic PCR. C: KiPSCs as control; 1 and 8: two separate pools of puromycin resistant colonies from two experiments; 2-7 and 9-14: six individual puromycin resistant colonies picked and expanded from each of two separate experiments. Copy number was calculated based on a standard curve using viral vector DNA as described previously (Sastry et al., 2002). (C) Immunofluorescence staining of KiPSCs and KiPSCs-SL with antibodies to pluripotency markers TRA-1-60 and TRA-1-81, and DAPI for nuclei. (D) Alcian Blue staining of high density cell mass (HDCM) from KiPSCs and KiPSCs-SL after three weeks of differentiation in the presence or absence of TGF $\beta$ stimulation. (E) Immunostaining (DAB) detected the expression of collagen II expression in HDCM from KiPSCs-SL with or without TGF $\beta$ stimulation for three weeks. (F) Changes in luciferase activity were comparable with Col II mRNA expression levels changes after one week of chondrocyte differentiation from KiPSCs-SL. Upper panel: qPCR analysis of the expression of Col II mRNA levels of HDCM in the presence or absence of TGF $\beta$ stimulation at indicated time points. Expression was normalized to samples without TGF $\beta$ stimulation at day 1; Lower panel: luciferase activity of HDCM with or without TGF $\beta$ stimulation. Expression was normalized to samples without TGF $\beta$ stimulation at day 7. Data represent the relative fold change (Mean \pm S.D.) from three independent experiments in duplicate. 
stimulation using a HDCM culture system. Alcian blue staining confirmed that KiPSCs-SL and KiPSCs have similar chondrocyte differentiation potential (Fig. 1D). Immunocytochemistry staining using a specific anti-collagen II antibody further showed that KiPSCs-SL could differentiate into mature chondrocytes (Fig. 1E).

Compound screening platforms strongly favor cell systems that do not require more than one week of culture, preferably less. However whether our KiPSCs-SL started to differentiate into chondrocytes in a detectable manner within one week was unknown. To answer this question, we performed chondrogenic differentiation with KiPSCs-SL in a shorter time-frame. QPCR analysis showed that the expression of Col II mRNA levels of KiPSCs-SL with TGF $\beta 1$ stimulation continued to increase and was about 11 fold higher at day 7 compared with day 1 . Though KiPSCs-SL without TGF $\beta 1$ also has increased expression of Col II mRNA, the Col II mRNA level was lower, showing only a 4-fold increase at day 7 compared with day 1 (Fig. 1F). The significant increase of Col II mRNA level with or without TGF $\beta 1$ indicated that cells started to differentiate into chondrocytes within one week of differentiation and that TGF $\beta 1$ can further promote this process. We then tested the luciferase activity of KiPSCs-SL under the same chondrogenic conditions. Similarly, luciferase activity also showed an approximate $3-4$ folds increase in cells differentiated with TGF $\beta 1$ stimulation compared to cells without TGF $\beta 1$ (Fig. 1F and 1G).

These results collectively demonstrate the successful generation of a chondrogenic reporter line from KiPSCs. KiPSCs-SL not only preserved the self-renewal and pluripotency characteristics of iPSCs, but could also faithfully represent endogenous Col II changes after chondrogenic differentiation. Furthermore, these data supported the feasibility of setting up and completing an automated screening assay within one week.

\section{Directed differentiation of KiPSCs-SL into chondrocytes in a 96-well format}

Screening platforms employ robotics and automated liquid handling systems that are designed for multi-well plates. Thus we sought to develop a modified chondrocyte differentiation protocol that did not rely on EB formation and 3D culture and could be performed largely in 96-well plates, which is the minimum miniaturization acceptable with these systems. Previously reported directed chondrogenesis protocols require changes of culture substrates during differentiation (Oldershaw et al., 2010). To streamline our protocol, we optimized our conditions to use only a Matrigel substrate. We obtained a specially prepared growth factor-free version of the stem cell medium mTeSR (custom mTeSR) as report (Ludwig et al., 2006), which enabled us to test a variety of cytokine combinations when differentiating KiPSCs and KiPSCs-SL into chondrocytes on Matrigel. These various trials led us to a simplified cytokine combination course during chondrocytes differentiation stage compared with previous one (Oldershaw et al., 2010), summarized in Table 1. As shown in Fig. 2A, 3 days of treatment with the combination of Wnt3a, Activin A, FGF2 and BMP4 largely increased the expression of the early mesoderm marker, T. However, the expression of the pluripotency genes OCT4 and NANOG were not yet downregulated by this timepoint. Following additional treatments with FGF2, BMP4 and Follistatin, the cells were further differentiated into the mesoderm lineage, as demonstrated by the induction of the later stage mesoderm markers KDR and SOX9 at day 7. By this timepoint, the

Table 1 Cytokine combination course for directed chondrogenesis

\begin{tabular}{|c|c|c|c|c|c|c|c|}
\hline Stage & Day & $\begin{array}{l}\text { WNT3A } \\
\text { (ng/mL) }\end{array}$ & $\begin{array}{l}\begin{array}{l}\text { Activin A } \\
\text { (ng/mL) }\end{array} \\
\end{array}$ & $\begin{array}{l}\text { FGF2 } \\
\text { (ng/mL) }\end{array}$ & $\begin{array}{l}\text { BMP4 } \\
\text { (ng/mL) }\end{array}$ & $\begin{array}{l}\text { Follistatin } \\
(\mathrm{ng} / \mathrm{mL})\end{array}$ & $\begin{array}{l}\text { GDF5 } \\
\text { (ng/mL) }\end{array}$ \\
\hline \multirow{7}{*}{$\begin{array}{l}\text { Stage I: Mesoderm } \\
\text { differentiation }\end{array}$} & 1 & 25 & 50 & & & & \\
\hline & 2 & 25 & 25 & 20 & & & \\
\hline & 3 & 25 & 10 & 20 & 40 & & \\
\hline & 4 & & & 20 & 40 & 100 & \\
\hline & 5 & & & 20 & 40 & 100 & \\
\hline & 6 & & & 20 & 40 & 100 & \\
\hline & 7 & & & 20 & 40 & 100 & \\
\hline \multirow{7}{*}{$\begin{array}{l}\text { Stage II: Chondrocyte } \\
\text { differentiation }\end{array}$} & \multicolumn{7}{|c|}{ Split and replate } \\
\hline & 8 & & & 20 & 40 & & \\
\hline & 9 & & & & & & 40 \\
\hline & 10 & & & & & & 40 \\
\hline & 11 & & & & & & 40 \\
\hline & 12 & & & & & & 40 \\
\hline & 13 & & & & & & 40 \\
\hline
\end{tabular}

The culture medium was changed daily with growth factor free (custom) mTeSR supplemented with the indicated cytokines. 
A
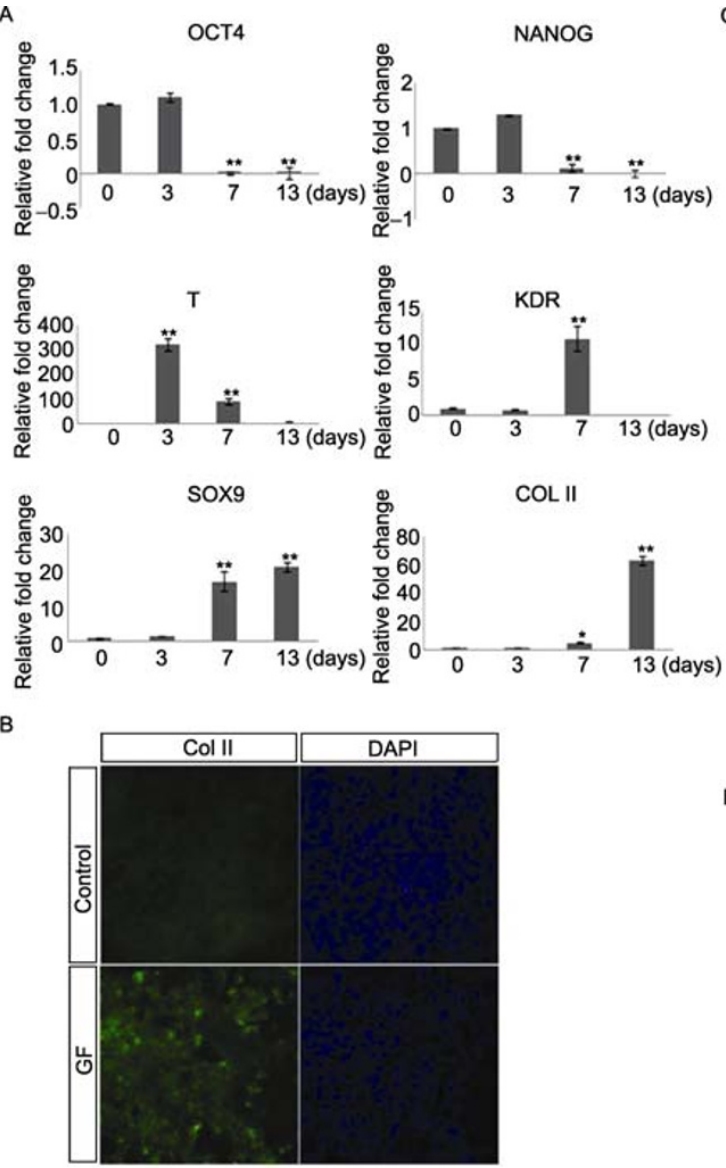

E

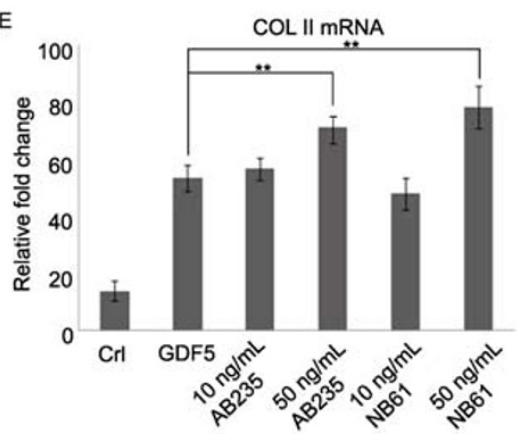

NANOG
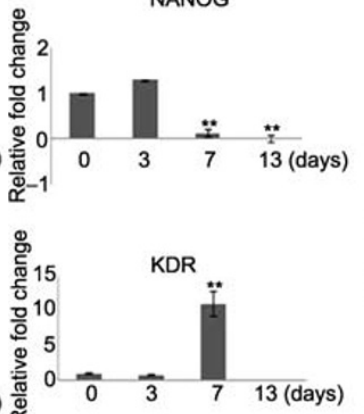

C
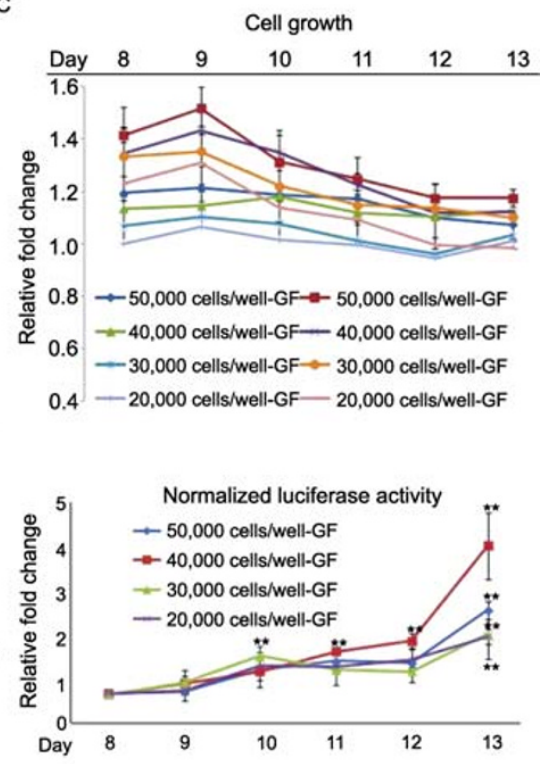

D
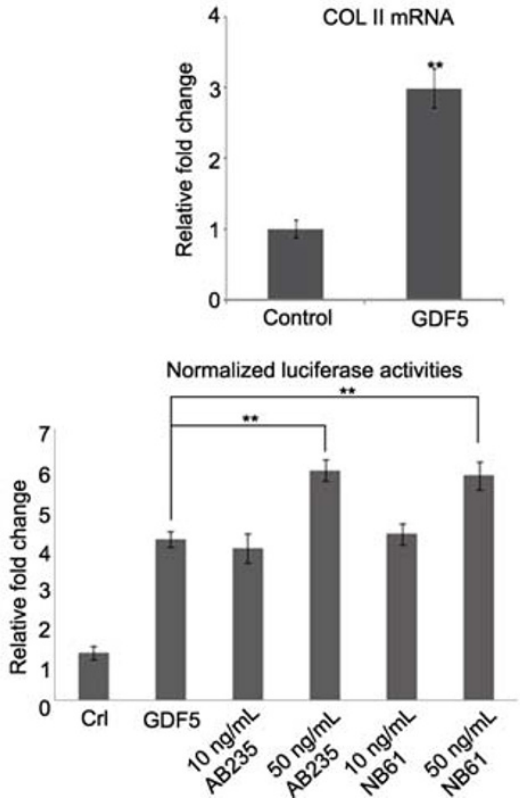

Figure 2. Directed differentiation of KiPSCs-SL into chondrocytes. (A) qPCR analysis for expression at indicated time points of selected pluripotent, mesoderm and chondrocyte genes during directed chondrogenesis. Expression was normalized to levels detected in KiPSCs at day 0. Data represents the relative fold change (Mean \pm S.D.) from three independent experiments in duplicate. (B) Immunofluorescence of Col II expression in cells at the end stage of chondrogenic differentiation; Control: custom mTeSR without growth factors, GF: custom mTeSR with added growth factors. (C) Chondrogenesis of KiPSCs-SL in a 96-well format. Upper panel: Cell Growth—cell numbers were normalized to 20,000 cells/well treated with custom mTeSR at day 8. Lower panel: Luciferase activity in each well was normalized to its corresponding cell number/ time point. Normalized luciferase activities were compared between different treatments in the same seeding density groups as relative fold change. Data represent relative fold change (Mean \pm S.D.) from three independent experiments in triplicate. (D) qPCR analysis of Col II mRNA expression of 40,000 cells/well group at the end stage of chondrogenesis (with and without GDF5). Data represent relative fold change (Mean \pm S.D.) from three independent experiments in triplicate. (E) Two synthesized compounds promote iPSCs chondrogenesis compared to GDF5 in a 96-well plate format. Left panel: GPCR analysis of the expression of Col II mRNA at the end stage of chondrogenesis after indicated treatments. Expression was normalized to levels in KiPSCs. Both samples treated with GDF5 or compounds expressed higher level of Col II mRNA compared to control with $P<0.01$. Right panel: luciferase activities at the end stage of chondrogenesis following indicated treatments. Luciferase activity was normalized to its corresponding cell number. Normalized luciferase activities were compared between different treatments as indicated. Both samples treated with GDF5 or compounds expressed higher luciferase activities compared to control with $P<0.01$. Data represents relative fold change (Mean \pm S.D.) from three independent experiments in triplicate. 
expression of OCT4 and NANOG were barely detectable. Though the expression of $\mathrm{T}$ dropped dramatically from day 3 to day 7 , it was still maintained at a relatively high level compared to undifferentiated cells. Finally, we used GDF5, a cytokine that belongs to the TGF $\beta 1$ family and is involved in chondrogenesis (Francis-West et al., 1999), to stimulate the cells to differentiate into mature chondrocytes. At this end stage (day 13), the expression of Col II was dramatically increased. Immunofluorescence analysis demonstrated that the protein level of Col II is also significantly induced (Fig. 2B). The combined changes in mRNA expression of NANOG, OCT4, T, KDR, SOX9 and COL II parallel those found in a previously reported differentiation protocol (Oldershaw et al., 2010). Thus, these collective data demonstrate that our newly modified differentiation protocol successfully directed chondrogenesis from KiPSC.

To further adapt our protocol to make it suitable for screening, we tested conditions to enable differentiation to occur in a 96-well format. After 7 days differentiating KiPSCs-SL into a mesoderm stage, we replated the cells into Matrigel coated 96-well plates and continued through the remaining phase of the differentiation protocol. We assayed cell ranges from 20,000 to 50,000 cells/well in triplicate, and collected the samples for both luciferase analysis and cell growth analysis each day, for 6 days. The basic medium, custom mTeSR, that contained no growth factor supplements, served as a control. Both wells seeded with different cell densities and different treatments formed compact cell monolayers one day after re-plating (Fig. 2C). There were no significant changes in cell number in either group treated with custom mTeSR alone for the following 6 days. However, the groups treated with growth factors have more cells than those treated with custom mTeSR during the first two days after plating into 96 well plates. Then the groups with growth factor continued to decrease in cell number regardless of the initial plating density. This phenomenon is consistent with previous reports describing that, while pre-chondrocytes group together, non-chondrogenic cells die off during the period allowed for differentiation (Oldershaw et al., 2010). Conversely, the luciferase activities of the groups with growth factors were higher than that of the groups treated only with custom mTeSR by 3 days after replating, and continued to increase. Although the 40,000 cells/well group showed no obvious increase in luciferase activity at earlier timepoints, this group showed the most pronounced increase in luciferase activity at the end of differentiation. To confirm our reporter line results, we performed qPCR analysis with RNA extracted from cells that had been plated and differentiated at a density of 40,000 cells/well in 96-well plate (Fig. 2D). The inductions of Col II mRNA levels were comparable with the observed increases in luciferase activity. These results show that we can differentiate KiPSCs-SL into chondrocytes in 96-well plate format, enabling a streamlined differentiation protocol that could be performed on an automated platform.
AB235 and NB61, two recently described novel compounds, are chimeric ligands of Activin/BMP2 (Allendorph et al., 2011). Since Activin and BMP2 can promote chondrogenesis in vitro and in vivo (Jiang et al., 1993; Majumdar et al., 2001), these chimeras should have chondrogenic potential. Here we used these two compounds to further validate our 96-well chondrogenic screening format. We tested each independently at two different doses and compared the effects to cells treated with GDF5. As shown in Fig. 2E, each compound is able to increase luciferase activity to a level comparable with GDF5. Interestingly, higher concentrations of each of these two compounds were able to improve chondrogenic differentiation compared to GDF5 (Fig. 2E). These luciferase reporter results were confirmed by qPCR analysis of Col II mRNA expression (Fig. 2E). Together, these findings demonstrate the successful generation of a cellular system that could be used to screen for novel regulators of chondrogenesis using an automated compound testing platform.

\section{DISCUSSION}

Recent reports have used hMSCs to screen for compounds that promote chondrogenesis (Huang et al., 2008; Johnson et al., 2012). However, due to variations between donors, limitations in expanding the hMSC populations, and cost (Siddappa et al., 2007), these cells were not ideal. We aimed to identify alternative cell sources which had unlimited expansion ability and chondrogenic ability. For these reasons, human iPSCs should be good cell sources for drug screening. However, each type of screen will need to incorporate methodologies that enable iPSCs to be translated successfully onto an automated platform. In each case, attention must be given to developing a protocol that is not only simple to execute (e.g. limited medium changes, the use of a single matrix throughout the assay), but that can also be performed in a miniaturized, multi-well format, and be completed in a short timeframe with clear, simple and reproducible endpoint readouts.

The classical chondrogenesis protocol established for hESCs is too time consuming and labor intensive for such a platform. Thus, we first generated a hiPSC chondrocyte reporter line to provide a simple endpoint readout, and then used this tool to develop a streamlined short protocol compatible with use in a 96-well format. Our new protocol enables chondrogenesis from pluripotent stem cells using only Matrigel coating as a matrix and growth factor-free mTeSR as basic medium so that the added growth factors can be controlled as necessary, resulting in a significantly simplified protocol from previous reports. Our protocol not only eliminates the need for EB formation and 3D culture, but can also be completed in a short timeframe. The ability to translate this approach into a drug-screening platform was validated by analyzing two novel compounds that demonstrated positive effects on chondrogenic potential. This study thus provides a 
specific and validated methodology that can be used in future screening efforts to identify novel small molecules active in promoting chondrogenesis.

\section{MATERIALS AND METHODS}

\section{Materials}

TGF- $\beta$ GDF5, BMP4, FGF2, Follistatin, Activin A are from Peprotech; WNT3A is from BD Biosciences; pyruvate, L-Proline, L-Ascorbic Acid, Dexamethasone are from Sigma; DMEM/F12, DMEM, L-glutamine, Penicillin/Streptomycin are from InVitrogen.

\section{Chondrocyte differentiation through embryoid body (EB) formation}

Chondrocyte differentiation from KiPSCs through EB formation was performed as previously described (Koay et al., 2007; Toh et al., 2007). Briefly, KiPSCs and KiPSCs-SL were cultured in Matrigel (BD Biosciences) with mTeSR medium (StemCell Technologies). The iPSC colonies were detached with Dispase, resuspended in EB medium (DMEM/F12 medium supplemented with 10\% FBS (Atlanta Biologicals), $0.1 \mathrm{mmol} / \mathrm{L}$ non-essential amino acids, $0.5 \mathrm{mmol} / \mathrm{L}$ L-glutamine, and $100 \mu \mathrm{mol} / \mathrm{L} \beta$-mercaptoethanol) and cultured in low attachment T-25 flasks for 7 days. EBs were collected and dispersed into single cells with TrypLE (InVitrogen), $3 \times 10^{5}$ cells per $20 \mu \mathrm{L} \mathrm{EB}$ medium were plated as drops in the center of one well of 12-well plate, and the plates were placed in an incubator $\left(37^{\circ} \mathrm{C}, 5 \% \mathrm{CO}_{2}\right)$ for $2 \mathrm{~h}$. Following the incubation period, $1 \mathrm{~mL}$ of EB medium was carefully added to each well and incubated overnight. The next day, the medium was changed to chondrogenesis medium (DMEM with 1 $\mathrm{mmol} / \mathrm{L}$ pyruvate, $40 \mu \mathrm{g} / \mathrm{mL}$ L-Proline, $0.1 \mathrm{mmol} / \mathrm{L}$ non-essential amino acids, 1\% ITS (BD Sciences), $1 \mathrm{mg} / \mathrm{mL}$ BSA, $250 \mu \mathrm{mol} / \mathrm{L}$ L-Ascorbic Acid, $10 \mathrm{nmol} / \mathrm{L}$ Dexamethasone, $2 \mathrm{mmol} / \mathrm{L}$ L-Glutamine, $100 \mathrm{U} / \mathrm{mL} P / S$ and $10 \mu \mathrm{g} / \mathrm{mL}$ TGF- $\beta$ and maintained for one or three weeks as indicated.

\section{Reporter line generation}

All DNA fragments were obtained by PCR and first cloned into pcDNA3.1 to obtain the cassette as shown in Fig. 1A. The whole cassette was then sub-cloned into a p156 lentiviral vector. One $10-\mathrm{cm}$ dish of HEK 293T cells was transfected with this lentiviral vector together with the corresponding packaging plasmids (pMDL, pCMV_VSVG and pRSV REV) by Lipofectamine 2000 (InVitrogen). At 48-72 $\mathrm{h}$ after transfection, the supernatant of the cell culture were collected and filtered. Virus was concentrated with ultracentrifugation at $19400 \mathrm{rpm}, 4^{\circ} \mathrm{C}, 2$ hours and resuspended in $200 \mu \mathrm{L}$ PBS.

KiPSCs were digested with TrypLE into single cells after one hour pre-incubation with Y-27632 (Sigma). $10^{6}$ cells were suspended in $270 \mu \mathrm{L}$ PBS with $30 \mu \mathrm{L}$ virus and $\mathrm{Y}-27632$ at $37^{\circ} \mathrm{C}$ for one hour. Cells were centrifuged, re-suspended with $\mathrm{mTeSR}$ with $\mathrm{Y}-27632$, and plated into one well of a 6 -well plate overnight at $37^{\circ} \mathrm{C}$. At 3 days after infection, cells were treated with $4 \mu \mathrm{g} / \mathrm{mL}$ puromycin (InvivoGen) for one week to select for positive clones. Genomic DNA extraction and integration copy number were calculated using methods previously reported (Laird et al., 1991; Sastry et al., 2002).

\section{Directed differentiation of KiPSC into chondrocytes}

The stem cell growth medium was made in-house following the reported composition of mTeSR (Ludwig et al., 2006) with the direct substitution of the zebrafish FGF2 growth factor for the human FGF2 version and replacement of L-glutamine with Glutamax. For the differentiation studies, we used a version of this medium that had the growth factors removed. The stem cell culture and differentiation we describe was also validated in commercial mTeSR1 (StemCell Technologies) and a custom mTeSR1 that was made for this study.

KiPSCs or KiPSCs-SL cells were seeded on Matrigel with mTeSR. When the cells reached about $10 \%$ confluency, the medium was changed to growth factor free (custom) mTeSR containing the combination of cytokines as shown in Table 1 . After 7 days of treatment, cells were separated into single cells by TrypLE. Cells $\left(2.5 \times 10^{5} /\right.$ well $)$ were seeded into one well of a 24 -well plate, or at the cell density indicated for each well of a 96-well plate.

\section{Alcian Blue staining and immunostaining:}

4\% (final) paraformaldehyde in PBS was used to fix cells at room temperature (RT) for 10 min. Samples were washed with PBS twice and stained with $1 \%$ Alcian Blue in $3 \%$ Acetic acid solution in distilled water for $30 \mathrm{~min}$ at RT. The samples were washed with distilled water until no further blue color eluted. For immunostaining, after fixation cells were exposed to $0.1 \%$ Triton $\mathrm{X}-100$ in PBS for 5 min then blocked with $10 \%$ goat serum in PBS for $1 \mathrm{~h}$ at RT. Samples were incubated with the appropriate primary antibody overnight at $4^{\circ} \mathrm{C}$. The next day samples were washed with $0.1 \%$ Tween-20 in PBS for 5 min and rinsed with PBS twice. The samples were then incubated with the corresponding secondary antibodies (InVitrogen) for $1 \mathrm{~h}$ at RT followed by DAB (Roche) detection or fluorescence microscopy. Primary antibodies were obtained from the following sources. Abcam: anti-Nanog (ab21624), anti-Col II (ab3092); Millipore: anti-TRA-1-60 (MAB4360), anti-TRA-1-81 (MAB4381).

\section{qPCR analysis}

Total RNA was extracted using TRIzol (InVitrogen) followed by cDNA synthesis using iScript reverse transcription supermix (Bio-Rad). Quantitative PCR was performed using SsoAdvanced SYBR Green Supermix on a CFX384 Touch Real-Time PCR detection system (Bio-Rad). Primer sequences used were as previously reported (Oldershaw et al., 2010).

\section{Luciferase activity and cell growth assay}

Luciferase activity assay was performed in accordance with the manufacturer's instructions for the One-Glu ${ }^{\mathrm{TM}}$ Luciferase Assay System (Promega). Cell growth was analyzed using a CyQUANT Cell Proliferation Assay kit (InVitrogen). Data was measured with a Synergy $\mathrm{H} 1 \mathrm{Hybrid}$ reader (Biotek).

\section{Statistic analysis}

Experiments were repeated three times. All the statistical analyses were performed using Student's $t$-tests. $P<0.01$ was defined as 
statistically significant.

\section{ACKNOWLEDGEMENTS}

Work in the laboratory of JCIB was supported by grants from MINECO, Fundacion Cellex, CIBER, Sanofi, The Leona M. and Harry B. Helmsley Charitable Trust and the G. Harold and Leila Y. Mathers Charitable Foundation. We thank Dr. Travis Berggren and Margaret Lutz of the Salk Institute Stem Cell Core for their technical support and useful discussions. We also thank the other members of the Belmonte laboratory for helpful discussions. Special thanks to Athanasia D. Panopoulos for editing this manuscript and May Schwarz and Peter Schwarz for dedicated administrative support.

\section{ABBREVIATIONS}

Col II, type II collagen; DAB, 3,3'-diaminobenzidine; EB, embryoid body; FBS, fetal bovine serum; HDCM, high density cell mass; hESC, human embryonic stem cells; HMG, high mobility group; iPSC, induced pluripotent stem cell; KDR, kinase insert domain receptor; KiPSCs, keratinocyte iPSCs; MEF, mouse embryonic fibroblast; MSC, mesenchymal stem cell; OA, osteoarthritis; QPCR, quantitative PCR; RT, room temperature; SOX9, SRY-related HMG-box 9; SRY, sex-determine region $\mathrm{Y}$; T, Brachyury

\section{REFERENCES}

Aasen, T., Raya, A., Barrero, M.J., Garreta, E., Consiglio, A., Gonzalez, F., Vassena, R., Bilic, J., Pekarik, V., Tiscornia, G., et al. (2008). Efficient and rapid generation of induced pluripotent stem cells from human keratinocytes. Nat Biotech 26, 1276-1284.

Allendorph, G.P., Read, J.D., Kawakami, Y., Kelber, J.A., Isaacs, M.J., and Choe, S. (2011). Designer TGF $\beta$ superfamily ligands with diversified functionality. PLoS ONE 6, e26402.

Bulman, S.E., Barron, V., Coleman, C.M., and Barry, F. (2012). Enhancing the mesenchymal stem cell therapeutic response: cell localization and support for cartilage repair. Tissue Eng Part B Rev. (In Press).

Chanda, D., Kumar, S., and Ponnazhagan, S. (2010). Therapeutic potential of adult bone marrow-derived mesenchymal stem cells in diseases of the skeleton. Journal of Cell Biochem 111, 249-257.

Dowthwaite, G.P., Bishop, J.C., Redman, S.N., Khan, I.M., Rooney, P., Evans, D.J.R., Haughton, L., Bayram, Z., Boyer, S., Thomson, B., et al. (2004). The surface of articular cartilage contains a progenitor cell population. J Cell Sci 117, 889-897.

Eiges, R., Schuldiner, M., Drukker, M., Yanuka, O., Itskovitz-Eldor, J., and Benvenisty, N. (2001). Establishment of human embryonic stem cell-transfected clones carrying a marker for undifferentiated cells. Curr Biol 11, 514-518.

Ellis, J., and Bhatia, M. (2011). iPSC technology: platform for drug discovery. Clin Pharmacol Ther 89, 639-641.

Falanga, V., Iwamoto, S., Chartier, M., Yufit, T., Butmarc, J., kouttab, N., Shrayer, D., and Carson, P. (2007). Autologous bone marrow-derived cultured mesenchymal stem cells delivered in a fibrin spray accelerate healing in murine and human cutaneous wounds. Tissue Eng 13, 1299-1312.

Francis-West, P.H., Abdelfattah, A., Chen, P., Allen, C., Parish, J.,
Ladher, R., Allen, S., MacPherson, S., Luyten, F.P., and Archer, C.W. (1999). Mechanisms of GDF-5 action during skeletal development. Development 126, 1305-1315.

Goldring, M.B., and Goldring, S.R. (2007). Osteoarthritis. J Cell Physiol 213, 626-634.

Huang, A., Motlekar, N., Stein, A., Diamond, S., Shore, E., and Mauck, R. (2008). High-throughput screening for modulators of mesenchymal stem cell chondrogenesis. Ann Biomed Eng 36, 1909-1921.

Inoue, H., and Yamanaka, S. (2011). The use of induced pluripotent stem cells in drug development. Clin Pharmacol Ther 89, 655-661.

Jiang, T.X., Yi, J.R., Ying, S.Y., and Chuong, C.M. (1993). Activin enhances chondrogenesis of limb bud cells: stimulation of precartilaginous mesenchymal condensations and expression of NCAM. Dev Biol 155, 545-557.

Johnson, K., Zhu, S., Tremblay, M.S., Payette, J.N., Wang, J., Bouchez, L.C., Meeusen, S., Althage, A., Cho, C.Y., Wu, X., et al. (2012). A stem cell-based approach to cartilage repair. Science 336, 717-721.

Koay, E.J., Hoben, G.M.B., and Athanasiou, K.A. (2007). Tissue engineering with chondrogenically differentiated human embryonic stem cells. Stem Cells 25, 2183-2190.

Koelling, S., Kruegel, J., Irmer, M., Path, J.R., Sadowski, B., Miro, X., and Miosge, N. (2009). Migratory chondrogenic progenitor cells from repair tissue during the later stages of human osteoarthritis. Cell Stem Cell 4, 324-335.

Kuettner, K.E. (1992). Biochemistry of articular cartilage in health and disease. Clin Biochem 25, 155-163.

Laird, P.W., Zijderveld, A., Linders, K., Rudnicki, M.A., Rudolf, J., and Berns, A. (1991). Simplified mammalian DNA isolation procedure. Nucleic Acids Res 19, 4293.

Liew, C.G., Draper, J.S., Walsh, J., Moore, H., and Andrews, P.W. (2007). Transient and stable transgene expression in human embryonic stem cells. Stem cells 25, 1521-1528.

Ludwig, T.E., Bergendahl, V., Levenstein, M.E., Yu, J., Probasco, M.D., and Thomson, J.A. (2006). Feeder-independent culture of human embryonic stem cells. Nat Meth 3, 637-646.

Lohmander, L.S., and Roos, E.M. (2007). Clinical update: treating osteoarthritis. The Lancet 370, 2082-2084.

MacArthur, C.C., Xue, h., Hoof, D.V., Lieu, P.T., Dudas, M., Fontes, A., Swistowski, A., Touboul, T., Seerke, R., Laurent, L.C., et al. (2012). Chromatin insulator elements block transgene silencing in engineered human embryonic stem cell lines at a defined chromosome 13 locus. Stem Cells Dev 21, 191-205.

Majumdar, M.K., Wang, E., and Morris, E.A. (2001). BMP-2 and BMP-9 promotes chondrogenic differentiation of human multipotential mesenchymal cells and overcomes the inhibitory effect of IL-1. J Cell Physiol 189, 275-284.

Oldershaw, R.A., Baxter, M.A., Lowe, E.T., Bates, N., Grady, L.M., Soncin, F., Brison, D.R., Hardingham, T.E., and Kimber, S.J. (2010). Directed differentiation of human embryonic stem cells toward chondrocytes. Nat Biotech 28, 1187-1194.

Oreffo, R., Cooper, C., Mason, C., and Clements, M. (2005). Mesenchymal stem cells. Stem Cell Rev Rep 1, 169-178.

Pridie, K.H. (1955). The development and nature of osteroarthritis of the hip joint. Rheumatism 11, 2-7. 
Sastry, L., Johnson, T., J Hobson, M., B, S., and K, C. (2002). Titering lentiviral vectors: comparison of DNA, RNA and marker expression methods. Gene Ther 9, 1155-1162.

Siddappa, R., Licht, R., van Blitterswijk, C., and de Boer, J. (2007). Donor variation and loss of multipotency during in vitro expansion of human mesenchymal stem cells for bone tissue engineering. $J$ Orthop Res 25, 1029-1041.

Smith, G.D., Knutsen, G., and Richardson, J.B. (2005). A clinical review of cartilage repair techniques. J Bone Joint Surg $\mathrm{Br} 87$, $445-449$

Steinwaerder, D.S., and Lieber, A. (2000). Insulation from viral transcriptional regulatory elements improves inducible transgene expression from adenovirus vectors in vitro and in vivo. Gene Ther 7, 556-567.

Takahashi, K., Tanabe, K., Ohnuki, M., Narita, M., Ichisaka, T., Tomoda, K., and Yamanaka, S. (2007). Induction of pluripotent stem cells from adult human fibroblasts by defined factors. Cell 131, 861-872.

Toh, W.S., Yang, Z., Liu, H., Heng, B.C., Lee, E.H., and Cao, T. (2007). Effects of culture conditions and bone morphogenetic protein 2 on extent of chondrogenesis from human embryonic stem cells. Stem Cells 25, 950-960.

William, R.J., and Harnly, H.W. (2007). Microfrature: indications, technique, and results. Instructional Course Lectures 56, 419-428.

Woolf, A.D., and Pfleger, B. (2003). Burden of major musculoskeletal conditions. Bulletin WHO, 646-656.

Yang, H.S., La, W.G., Bhang, S.H., Kim, H.J., Im, G.I., Lee, H., Park, J.H., and Kim, B.S. (2011). Hyaline cartilage regeneration by combined therapy of microfracture and long-term bone morphogenetic protein-2 delivery. Tissue Eng Part A 17, 1809-1818.

Yu, J., Vodyanik, M.A., Smuga-Otto, K., Antosiewicz-Bourget, J., Frane, J.L., Tian, S., Nie, J., Jonsdottir, G.A., Ruotti, V., Stewart, $R$., et al. (2007). Induced pluripotent stem cell lines derived from human somatic cells. Science 318, 1917-1920.

Zhou, G., Lefebvre, V., Zhang, Z., Eberspaecher, H., and de Crombrugghe, B. (1998). Three high mobility group-like sequences within a 48-base pair enhancer of the Col2a1 gene are required for cartilage-specific expression in vivo. J Biol Chem 273, 14989-14997. 